\title{
ACOMPANHAMENTO POLISSONOGRÃFICO DAS APNÉIAS DO SONO APÓS UVULOPALATOFARINGOPLASTIA
}

\author{
RUBENS REIMAO * \\ HELIO LEMMI ** \\ HAGOP AKISKAL *** \\ EDWIN COCKE ****
}

Traqueostomia é o tratamento cirúrgico mais utilizado atualmente para correção da apnéia do sono tipo obstrutiva. Sua eficácia imediata e a médio prazo é comprovada mas suas potenciais complicações, dificuldade de manutenção e as barreiras psicossociais que levanta levaram a procura de outras formas terapêuticas. A uvulopalatofaringoplastia (UPFP), que visa retirar tecido redundante do orofaringe facilitando a passagem aérea em pacientes com apnéia do sono foi introduzida recentemente por Fujita e col.2, e seu uso tem se difundido mas, suas conseqüências a médio e longo prazo são ainda insuficientemente conhecidas 1 ,4.

Relatamos as alterações polissonográficas observadas a médio prazo em pacientes submetidos a UPFP.

\section{CASUfSTICA E METODOLOGIA}

Foram avaliados 12 pacientes (11 homens; 1 mulher) submetidos a UPFP por apresentarem apnéia do sono tipo obstrutiva. A média etária fol 47,9 anos e as idades variavam de 29 a 64 anos. Apenas um caso tinha peso na faixa normal; nos demais estava acima. Dez pacientes eram de cor branca e dois, negros. O diagnóstico foi baseado nas consultas clínica, neurológica, psiquiátrica e otolaringológica, e no traçado polissonografico. A investigação clínica incluía testes de funções pulmonares e monitorizaçăo cardiaca de 24 horas (Holter). Todos os pacientes fizeram novo traçado um a três meses após a cirurgia (média $=1,6$ meses) e este foi comparado ao pré-operatório. A UPFP fol a única cirurgia realizada em 7 casos. Nos 5 demais a UPFP fol acompanhada de traqueostomia temporária visando fornecer alívio imediato dos sintomas. Tais pacientes foram mantidos com canula fechada durante o dia e aberta apenas ao dormir, exceto por ocasião do registro polissonográfico quando a canula foi mantida fechada.

O traçado polissonográfico padronizado incluia eletrencefalograma (EEG), eletrocardiograma (FCG), eletromiograma dos músculos submentonianos e tibiais anteriores,

Trabalho do Department of Neurodiagnostics, Baptist Memorial Hospital e da University of Tennessee, Memphis, Tennessee, E.U.A.: *Neurologista; ** Clinical Professor of Neurology; *** Professor of Psychiatry and Associate Professor of Pharmacology; **** Clinical Professor of Otolaryngology. 
deteç̧ão de tluxo aéreo por pares termoelétricos nasais e bucais, análise de esforço respiratório por pneumograma toráxico e abdominal e percentagem de saturação de oxigénio (SaO2). Obtivemos EEG nas derivações C3-A2 e C4-A1. ECG foi registrado na derivação V5. Comportamento e rufdos respiratórios foram observados durante toda a noite e gravados em video tape. As salas tinham isolamento acústico e temperatura mantida em torno de $23^{\circ} \mathrm{C}$. Os traçados foram julgados quanto aos estágios de sono pelos critérios de Rechtschaffen e col. (6). Utilizamos as definições de apnéía referidas por Guilleminault e col. (3) e duração minima de 6 s. Indice de apnéia (IA), ou número de apnéias obstrutivas ou mistas por hora de sono (3), fol considerado normal quando $<5$ no sexo masculino $e<1$ no feminino. Despertares completos foram incluídos apenas se de duração $>30 \mathrm{~s}$. Latência do sono REM fol caracterizada como o tempo entre o início do estágio 2 e o início do sono REM, considerando-se anormais valores abaixo de $70 \mathrm{~min}$.

\section{RESULTADOS}

A queixa de sonolência excessiva diurna, referida por todos os pacientes antes da cirurgia, fol reduzida parcialmente em 4 casos e totalmente em 8, após UPFP. Embora sempre se aconselhasse perda de peso, apenas 4 casos tiveram redução $\geqslant 5 \mathrm{~kg}$ e os demais permaneceram estáveis.

Tempo total de sono, eficiencia do sono e número de despertares completos foram semelhantes em ambas avaliações (Tabela 1). A média de latência do sono também pouco se alterou após a cirurgia mas apenas 4 casos tiveram latências maiores que 5 min no pré enquanto 8 tiveram este índice no pós-operatório. A média de latência REM foi acentuadamente maior após a cirurgia. Latência REM $<70 \mathrm{~min}$ foi vista em 7 casos no pré e apenas em um no pós-operatório. A duração dos estágios de sono como percentagem do tempo total de sono teve médias próximas nas duas noites. Intretanto, deve-se mencionar que dois pacientes não atingiram estágio 3 na primeira noite e outros dois na segunda; o estágio 4 estava ausente em 9 casos na primeira noite e em 6 na segunda. Valores mínimos de $\mathrm{SaO2}$ estavam abaixo de $80 \%$ em 10

\begin{tabular}{lcc}
\hline & Antes da UPFP & Após a UPFP \\
\hline \hline Tempo total de sono (min) & 394,4 & 389,7 \\
Eficiência do sono (\%) & 92,4 & 88,3 \\
Latência do sono (min) & 5,4 & 6,4 \\
Latência REM (min) & 95,6 & 147,9 \\
Despertares completos & 3,7 & 3,9 \\
Erstágio 1 (\%) & 6,0 & 6,5 \\
Estágio 2 (\%) & 66,9 & 65,1 \\
Estágio 3 (\%) & 8,5 & 9,9 \\
Estágio 4 (\%) & 1,2 & 2,7 \\
Estágio REM (\%) & 17,0 & 15,5 \\
Indice de apnéia & 43,3 & 14,5 \\
\hline
\end{tabular}

Tabela 1 - Médias dos dados polissonograficos antes e apos uvulopalatofaringoplastia (UPFP) em 12 pacientes com apnéia do sono tipo obstrutiva. 
casos no pré e em 4 no pós-operatório. Alterações do ECG relacionadas às apnéias estavam presentes em 8 casos antes da cirurgia e em 6 após, sendo a alternancia entre bradicardia e taquicardia a cada apnéia e as contraçóes ventriculares prematuras as mais encontradas. O IA diminuiu em todos os casos após UPFP mas apenas 4 atingiram valores normais. Esta normalização não se relacionou a qualquer dos fatores analisados, incluindo: idade, sexo, peso, intervalo entre as avaliações, melhora da sonolência, traqueostomia, tempo total de sono, eficiência do sono, latência do sono, latência REM, número de despertares, distribuição dos estágios de sono, $\mathrm{SaO2}$ e ECG.

\section{COMENTARIOS}

$\mathrm{Na}$ maior parte dos casos não se observa qualquer alteração do orofaringe em pacientes com apnéia do sono tipo obstrutiva mas, achados inespecificos podem ser relatados como úvula longa e com base larga, mucosa redundante formando pregas na parede posterior da faringe e no pilar posterior e aumento da base da língua. Estudos feitos durante as apnéias, com endoscopia e fluoroscopia evidenciam colabamento das paredes do orofaringe 5,11 e a eletromiografia revela hipotonia dos músculos constritores da faringe e dos músculos da língua 5,7, demonstrando não se tratar de diminuição do lúmen por contração muscular. Esta obstrução que ocorre durante a inspiração é favorecida pelo efeito de sucção somado a hipotonia própria do sono, a qual se acentua no estágio REM. Baseada na necessidade de facilitar a passagem aérea, a UPFP consiste da retirada da úvula, borda posterior do pálato mole e da mucosa redundante das paredes faríngeas e do pilar posterior 2 . Em nossa casuística, baixos índices de tempo total de sono e eficiência do sono assim como grande número de despertares completos nas avaliações pré e pós-operatórias refletem a rotura dos padrões normais provocada pelas apnéias.

Após UPFP houve normalização da latência do sono REM. Atribuímos esta melhora ao fato de levarem as apnéias a superficialização freqüente do sono impedindo a ocorrência ininterrupta do estágio REM. Tal privação parcial do sono REM leva à tendência de surgimento precoce deste estágio a cada noite. A ocorrência de sono REM com menor número de interrupções no pós-operatório possibilita redução da pressão de aparecimento e normalização de sua latência. As apnéias levam também a superficialização do sono, diminuindo tanto a ocorrência de estágio 4 como a duração dos estágios 3 e 4 verificadas em nossa casuística, e que foram minimizadas após UPFP. A manutenção do tempo dispendido nos estágios 1 e 2 após a cirurgia em nossa amostra difere dos dados obtidos por Fujita e col.2 que mostraram redução do estágio 1 e aumento do 2 no pós-operatório. Esta diferença talvez se deva a heterogeneidade do tempo de seguimento daqueles autores.

No grupo aqui relatado, a UPFP levou a redução da sonolência excessiva em todos os casos, melhora dos níveis de $\mathrm{SaO} 2$ em mais da metade dos pacientes e normalização do IA em um terço. Esta redução acentuada da sonolência, subjetiva com menor mudança dos índices objetivos, concorda com dados de outras publicações 8,9,10. Baseados em nosso seguimento polissonográfico a 
médio prazo podemos concluir que a UPPFP pode ser cirurgia alternativa a traqueostomia em alguns casos. Consideramos que se destina particularmente àqueles com menor comprometimento, nos quais o alivio das manifestações cardiopulmonares pode ser gradual, e para os quais a traqueostomia pode ser realizada posteriormente se a UPFP não trouxer resultados satisfatórios.

\section{RESUMO}

Uvulopalatofaringoplastia (UPFP) foi realizada em 12 pacientes com apnéia do sono tipo obstrutiva. Comparamos aqui dados clínicos e traçados polissonográficos pré-operatórios aos realizados de um a três meses após a cirurgia. Sonolência excessiva diurna foi reduzida parcialmente em 4 casos e totalmente em 8. Tempo total de sono, eficiência do sono, número de despertares e latência do sono mantiveram-se constantes. A latência REM foi acentuadamente maior após a cirurgia. A distribuição dos estágios de sono foi semelhante nas duas avaliações exceto pela ausência de estágio 4 verificada em menor número de casos no pós-operatório. Houve melhora da $\mathrm{SaO2}$, encontrando-se valores abaixo de $80 \%$ em 10 pacientes no pré e em 4 no pós-operatório. Os índices de apnéia reduziram-se em todos os casos, mas em apenas 4 atingiram valores normais.

\section{SUMMARY}

Polysomnographic follow-up of sleep apneas after uvulopalatopharyngoplasty.

Uvulopalatopharyngoplasty (UPPP) was performed in 12 patients with obstructive sleep apnea. Pre-surgical clinical and polysomnographic data were compared with those one to three months after surgery. Excessive daytime sleepiness was partially improved in 4 cases and completely abolished in 8 . Total sleep time, sleep efficiency, number of arousals and sleep latency were similar in both evaluations. REM latency to stage 2 markedly increased on the follow-up. Stage distributions were similar in both nights except for stage 4 which was more frequently absent on the first recording. SaO2 levels below $80 \%$ were seen in 10 cases before UPPP and in 4 after it. Apnea index decreased in all cases but it reached normal levels only in 4 .

\section{REFERENCIAS}

1. BOROWIECKI, B.B. \& SASSIN, J.F. - Surgical treatment of sleep apnea. Arch. Otolaryngol. 109:508, 1983.

2. FUJITA, S.; CONWAY, W.; ZORICK, R. \& ROTH, T. - Surgical correction of anatomical abnormalities in obstructive sleep apnea syndrome: uvulopalatopharyngoplasty. Otolaryngol. Head Neck Surg. 89:923, 1981.

3. GUILLFMINAULT, C.; HOED, J.V.D. \& MITLER, M.M. - Clinical overview of the sleep apnea syndromes. In C. Guilleminault \& W.C. Dement (eds.) - Sleep Apnea Syndromes. Alan R. Liss, New York, 1978, pg. 1.

4. HERNANDEZ, S.F. - Palatopharyngoplasty for the obstructive sleep apnea syndrome: technique and preliminary report of results in ten patients. Amer. J. Otolaryngol. 3:229, 1982. 
5. HILL, N.W.; GUILLEMINAULT, C. \& SIMMONS, R.B. - F'iber-optic and FMG studies in hypersomnia-sleep apnea syndrome. In C. Guilleminault \& W.C. Dement (eds.) - Sleep Apnea Syndromes. Alan R. Liss, New York, 1978, pg. 249.

6. RECHTSCHAFFEN, A. \& KALES, A. - A Manual of Standardized Terminology, Techniques and Scoring for Sleep Stages of Human Subjects. Brain Information Service/Brain Research Institute. University of California, Los Angeles, 1968.

7. REMMERS, J.S.; GROOT, W.J.; SAUERLAND, E.K. \& ANCH, A.M. - Pathogenesis of upper airway occlusion during sleep. J. appl. Physiol. 44:931, 1978.

8. SILVESTRI, R.; GUILLEMINAULT, C. \& SIMMONS, F.B. - Palatopharyngoplasty in the treatment of obstructive sleep apnea patients. In Guilleminault \& E. Lugaresi (eds.) - Sleep/Wake Disorders: Natural History, Epidemiology and Long-Term Evolution. Raven Press, New York, 1983, pg. 163.

9. SIMMONS, F.B.; GUILLEMINAULT, C. \& SILVESTRI, R. - Snoring and some obstructive sleep apnea can be cured by oropharyngeal surgery. Arch. Otolaryngol. 109:503, 1983.

10. THORNPY, M.J.; SHER, A. \& SPIFLMAN, A.J. - Uvulopalatopharyngoplasty (UPPP) for obstructive sleep apnea: II. Farly results. Sleep Res. 13:171, 1984.

11. WEITZMAN, E.D.; POLLAK, C.P. \& BOROWIFCK, B. - The hypersomnia-sleep apnea syndrome, site and mechanisms of upper airway obstruction. In C. Guilleminault \& W.C. Dement (eds.) - sleep Apnea Syndromes. Alan R. Liss, New York, 1978, pg. 235.

Sleep Disorders Center, Department of Neurodiagnostics, Baptist Memorial Hospital 899 Madison Avenue - Memphis, Tennessee, 38146 - U.S.A. 